\title{
Fractal adsorption characteristics of complex molecules on particles-A case study of dyes onto granular activated carbon (GAC)
}

\author{
Yili Wang ${ }^{\mathrm{a}, *}$, Hongwei Zhou ${ }^{\mathrm{b}}$, Fuling Yu ${ }^{\mathrm{a}}$, Baoyou Shi ${ }^{\mathrm{c}}$, Hongxiao Tang ${ }^{\mathrm{c}}$ \\ a Environmental Science, College of Natural Resource and Environment, The Key Laboratory for Silviculture and Conservation \\ of Ministry of Education and Beijing, Beijing Forestry University, Beijing 100083, China \\ ${ }^{\mathrm{b}}$ Institute of Rock Mechanics and Fractals, China University of Mining and Technology, Beijing 100083, China \\ c State Key Laboratory of Environmental Aquatic Chemistry, Research Center for Eco-Environmental Sciences, \\ Chinese Academy of Sciences, Beijing 100085, China
}

Received 25 December 2005; received in revised form 5 October 2006; accepted 26 November 2006

Available online 30 November 2006

\begin{abstract}
The isothermic and kinetic adsorption processes of three dyes onto granular activated carbon (GAC) were studied in this paper. Langmuir fractal equation was chosen to fit the adsorption isotherm data. The exponent $m$ in the equation is a function of surface fractal dimension $\left(D_{\mathrm{s}}\right)$ of particles and cross-section area $\left(\alpha_{0}\right)$ or radius $\left(r_{0}\right)$ of dye molecules, which can be approximately estimated according to the relation between the first order term of molecular connective index ${ }^{1} \chi$ and the total surface molecular area (TSA). Based on the modeling results, $D_{\mathrm{s}}$ of the GAC was estimated to be 2.59 , indicating the fractal adsorption characteristics of GAC.

To elucidate the adsorption kinetics of complex molecules onto fractal surfaces, the relationships of $m$, kinetic parameter $h$ and fractal spectral dimension $\left(d_{\mathrm{s}}\right)$ were developed, and the derivative relation of $d_{\mathrm{s}}$ and surface fractal dimension $D_{\mathrm{s}}$ was achieved. Adsorption kinetic results showed that the kinetic process had fractal-like characteristics. The instantaneous rate coefficient exhibited power-law relation with reaction time. Although $h$ was larger than one in this study, both the fractal spectral dimension $\left(d_{\mathrm{s}}\right)$ and the order of reaction could be obtained, and the corresponding $d_{\mathrm{s}}$ values were less than two. The effective reaction order $x$ was related to $d_{\mathrm{s}}, D_{\mathrm{s}}$ and adsorbate concentration, and it was found that the order of diffusion-controlled adsorption reaction was fractional in most cases. The $h$ or $d_{\mathrm{s}}$ values calculated here implied that the reciprocating shake was not always effective to eliminate the dimensional constraints.
\end{abstract}

(C) 2006 Elsevier B.V. All rights reserved.

Keywords: Fractal adsorption characteristics; GAC; Surface fractal dimension $D_{\mathrm{s}}$; Spectral dimension $d_{\mathrm{s}}$; Non-integer effective reaction order

\section{Introduction}

In natural water environments and engineered water treatment systems, solid particles are ubiquitous and their existence can affect the speciation distribution, transportation and transformation of many pollutants, such as heavy metals, inorganic nutrients and toxic organic matters. Solid particles can act as micro-reactors since a large number of physicochemical and biological reactions can happen on the solid-liquid microinterfaces. It is well known that adsorption of pollutants onto particle surface plays an important role in determining the fate of pollutants [1]. Therefore, adsorption has long been research

\footnotetext{
* Corresponding author. Tel.: +86 1062337917 ; fax: +86 1062341948

E-mail addresses: wangyilimail@126.com,wangyilinet@sina.com (Y. Wang).
}

topic in environmental science and engineering fields. Different models and mechanisms have been put forward to explain the adsorption behaviors under some specific conditions. However, the effect of particle geometrical morphology on adsorption is still not well understood [2].

The highly inhomogeneous surface structure of solid particles is difficult to characterize using simple geometry. Fractals, whose structures repeat themselves in all dimensions and on all length scales, are often used to depict the structure of solid particles and their surfaces [3-6]. Generally a rigid object with an infinitely compact interior has a mass fractal dimension $(D)$ of three. While the mass fractal dimensions of solid particles with irregular surface and pores are usually less than three. As to surface fractal dimension $\left(D_{\mathrm{s}}\right), D_{\mathrm{s}}$ of two means the surface is regular and smooth, while a higher $D_{\mathrm{s}}$ value indicates a greater wiggle and space-filling surface. If $D_{\mathrm{s}}$ value approaches to three, the surface is extremely irregular [7]. Douglas [8] has reported 


\section{Nomenclature}

$b \quad$ parameter related to the heat of adsorption in the fractal Langmuir adsorption isotherm equation in aqueous media

$C_{\mathrm{e}} \quad$ the equilibrium solute concentration;

$d_{\mathrm{s}} \quad$ spectral dimension

$d_{\mathrm{w}} \quad$ fractal dimension of random-walk route in fractal structure;

$D \quad$ typical fractal dimension representing the fractal structure of an object, mass dimension

$D_{\text {s }} \quad$ surface fractal dimension

$h$ parameter measuring the degree of local heterogeneity

$k \quad$ reaction rate coefficient

$K \quad$ a proportional coefficient

$L$ the average distance of a random walk after time $t$

$m \quad$ average number of surface binding sites covered by one adsorbate molecule

$n_{\mathrm{e}} \quad$ the side number

$N$ the total number of distinct lattice sites on particle surface

$N_{\mathrm{A}} \quad$ the total number of distinct lattice sites that is occupied by binding sites

$N_{\mathrm{A}}^{\prime} \quad$ the number of distinct lattice sites not covered by complex organic molecule at time $t$ complex organic molecule and the binding sites of particle surface

$P \quad$ the recurrence probability of a random walker after time $t$

$r_{0} \quad$ the radius of molecule gyration

$S$ the number of distinct lattice sites visited by randomly walking complex organic molecule

TSA total surface area of organic molecule

\section{Greek symbols}

$\alpha_{0} \quad$ the cross-sectional area of the adsorbate molecule

$\Gamma \quad$ the amount of solute adsorbed

$\delta_{i}, \delta_{j} \quad$ the valence of graph vertex of a side in the hydrogen-suppressed graph "area or radius versus $m$ " in $1 g-1 g$ scale

$\rho \quad$ the density of active groups on complex organic molecule

${ }^{1} \chi \quad$ the first order term of molecular connectivity index $p \quad$ the reaction probability between active groups of

$\lambda$ the regression line slope of the relation between

cle surfaces. When these models are used, it is usually assumed that the surface structure of adsorbent is uniform. However, all particle surfaces are actually heterogeneous, and can be characterized by the extent of irregularity or roughness. To take the surface heterogeneity into account, Avnir et al. [9], Pfeifer et al. [10], Fripiat et al. [11], Ma et al. [12] developed fractal BET equations, Kanô et al. [13] and Zhao et al. [14] developed fractal Langmuir and Freundlich equations.

In addition, Kopelman [15,17], Klymko and Kopelman [16] and Alexander and Orbach [18] found that classical reaction kinetics is not applicable when reactants are spatially constrained on microscopic level by walls, phase boundaries, or force fields. Many physical, chemical and biological reactions in real systems belong to those so-called "heterogeneous reactions". Usually, the fractal-like kinetics is used to characterize the heterogeneous reactions with spectral (or random-walk recurrence) dimension $d_{\mathrm{s}}$. Heterogeneous reactions have some unique features, such as fractal orders for elementary reactions, self-ordering and self-unmixing of reactants, and rate coefficients with temporal "memories". Generally, fractal spaces are ideal testing grounds for fractal-like reaction kinetics; however, reaction medium does not have to be a geometrical fractal in order to exhibit fractal kinetics.

It can be expected that adsorption processes involving solid-liquid micro-interfaces or boundaries could be of high heterogeneity. Theoretical studies of adsorption on fractal surfaces predicted the potential effect of surface fractal dimension on diffusion-limited adsorption kinetics [19,20]. The molecular diffusion has important influence on the adsorption reaction rate, especially for media with multi-pores. Kopelman [15] stated that in locally heterogeneous media, the diffusion-controlled reaction kinetics of bimolecular elementary reaction demonstrated fractal characteristics. Liu et al. [21] proposed that rock dissolution could also be a fractal-like process. Kinoshita et al. [22] considered that the adsorption of $\mathrm{Sr}^{2+}$ onto sandy rock is a fractal-like one too.

However, it has not yet been determined if the adsorption process of complex organic molecules (such as dyes) onto particle surfaces is fractal-like, and if the spectral dimension and the order of reaction can be obtained.

In this paper, the adsorption of three different dyes onto GAC was investigated. Langmuir fractal adsorption isotherm equations were adopted to fit the adsorption isotherm data. Furthermore, to elucidate the adsorption kinetics of complex molecules onto fractal particle surfaces, the relations of kinetic parameter $h$ with both fractal spectral dimension $d_{\text {s }}$ and surface fractal dimension $D_{\mathrm{s}}$ were firstly developed. In addition, the fractal-like kinetic characteristics of dye adsorption onto GAC were confirmed.

\section{Theory}

that adsorption of polymers on rough surfaces could be affected by surface fractal dimension.

Adsorption isotherms are widely employed in estimating the surface fractal dimensions of absorbent particles [9-14]. Langmuir, Freundlich and BET equations are among the most classical models to describe the adsorption of solutes onto parti-

\subsection{Fractal adsorption models for Langmuir and Freundlich equations}

The surfaces of environmental particles, such as activated carbon, sediments, granular sludge, are all irregular to some extent. 
An important characteristic of such surfaces is that the irregularity is similar at various resolution scales. Due to the irregularity of surfaces, it can be imagined that some surface binding sites could become unavailable for adsorption if the molecular size of the adsorbate is relatively large. This situation may be occurred for single-layer adsorption in water solution with low concentration of adsorbate. Under such condition, the interaction among the adsorbed molecules could be neglected. If $m$ is the average number of surface binding sites covered by one adsorbate molecule and among them, only $n^{\prime}$ sites are bound to the adsorbate molecule, there will be some surface sites are unable to be accessed by other molecules due to the space restriction.

Kanô et al. [13] and Zhao et al. [14] developed the fractal Langmuir adsorption isotherm equation in aqueous media as the following:

$\Gamma=\frac{\Gamma_{\mathrm{m}} C_{\mathrm{e}}^{1 / m}}{b^{m}+C_{\mathrm{e}}^{1 / m}}$

where $\Gamma$ and $\Gamma_{\mathrm{m}}$ represent the amount of solute adsorbed and the maximum monolayer adsorption capacity $\left(\mathrm{mg} \mathrm{g}^{-1}\right)$, respectively, $C_{\mathrm{e}}$ the equilibrium solute concentration $\left(\mathrm{mgl}^{-1}\right), b$ the parameter related to the heat of adsorption and proportional to the ratio between the adsorption reaction rate coefficient and the desorption reaction rate coefficient when adsorption reaches equilibrium [14] and $m$ is the average number of surface sites covered by one adsorbate molecule.

If $C_{\mathrm{e}}$ is very low, $b^{m}$ will be far greater than $C_{\mathrm{e}}^{1 / m}$, Eq. (1) can be transformed to

$\Gamma=\frac{\Gamma_{\mathrm{m}} C_{\mathrm{e}}^{1 / m}}{b^{m}}$

It is known that $m$ is a function of surface fractal dimension $D_{\mathrm{s}}$ in the following form [23,24]:

$m \propto a_{0}^{\left(D_{\mathrm{s}} / 2\right)-1}$ or $m \propto r_{0}^{D_{\mathrm{s}}-2}$

where $\alpha_{0}$ is the cross-sectional area of the adsorbate molecule and $r_{0}$ is the radius of molecule gyration. The logarithmic transformation of this equation and the subsequent regression of the relation of "area or radius versus $m$ " in $\lg -\lg$ scale can provide the value of $D_{\mathrm{s}}$ as

$D_{\mathrm{s}}=2 \lambda+2$

where $\lambda$ is the slope of regression line.

\subsection{Kinetic characteristics of fractal-like adsorption of complex molecules onto environmental particles}

It was observed that in most heterogeneous phases, the rate constant $k_{1}$ (or instantaneous rate coefficient) of reaction kinetics depends on reaction time $t[15,16]$ :

$k_{1}(t)=k_{0} t^{-h}$

where $k_{0}$ is a constant not dependent on time and $h$ is a parameter measuring the degree of local heterogeneity [16]. The rate constant and the order of reaction are associated with the spectral dimension $d_{\mathrm{s}}$.
The spectral dimension $d_{\mathrm{S}}$ for fractal objects is a second dimension (in addition to the typical fractal dimension $D$ representing the fractal structure of an object, mass dimension) defined by the recurrence probability $P$ of a random walker, that is, the probability $P$ of random walker to return to its origin after time $t: P \propto t^{d_{\mathrm{s}} / 2}$ [15]. Alexander and Orbach [18] proposed that the spectral dimension $d_{\mathrm{S}}$ could be used to describe the anomalous time or frequency scale properties of fracton (phonon on fractal) excited by vibration element in fractal medium (a microscopically heterogeneous medium). The $d_{\mathrm{s}}$ value means the degree of local heterogeneity. Increase of $d_{\mathrm{s}}$ value implies more local heterogeneous, more remarkable time-dependent rate coefficient.

Kopelman also noticed that $d_{\mathrm{s}} \leq D$ for all fractals. For a single reactant bimolecular reaction $(\mathrm{A}+\mathrm{A})$, there should be the following relation:

$h=1-\frac{d_{\mathrm{s}}}{2}$

and the order $x$ of this anomalous reaction under condition of diffusion-limitation can be obtained by [17]:

$x=1+\frac{2}{d_{\mathrm{s}}} \quad$ or $\quad x=1+(1-h)^{-1}$

Alexander and Orbach [18] pointed out that the spectral dimension of fractal objects could also be calculated by

$d_{\mathrm{s}}=\frac{2 D}{d_{\mathrm{w}}}$

where $d_{\mathrm{w}}$ represents fractal dimension of random-walk route in fractal structure, defined by the average distance $L$ of a random walk after time $t: t \propto L^{d_{\mathrm{w}}}$ [18], then $d_{\mathrm{s}}$ and $d_{\mathrm{w}}$ are parameters for vibration and diffusion kinetic processes, respectively. Therefore, Eq. (8) gives the important relations between static and kinetic properties of fractal structure.

Generally, complex organic molecules have some functional groups (active groups), which can interact with the surface binding sites on particle surface. The adsorption process can be regarded as a diffusion-controlled multi-group-site reaction in fractal media, similar to the multi-molecule reaction kinetic process. However, if the corresponding $h$ value is greater than one, the spectral dimension and the order of reaction cannot be obtained by using Eqs. (6) and (7).

Therefore, new equations should be developed to estimate the spectral dimension and the order of the diffusion-controlled multi-group-site reaction on fractal surface. During the adsorption of complex organic matter, one organic molecule could occupy some surface area larger than that containing equivalent amount of binding sites because of the irregularity of the surface structure. If each active group on complex organic molecule and each binding site on particle surface are taken as simple molecules, the diffusion-adsorption process between them can be viewed as a bimolecular elementary reaction as Kopelman proposed. Fig. 1 is the schematic illustration of such diffusion-adsorption process.

Kanô et al. [13] proposed a model for carbon surface: the carbon surface is stretched to an imaginary flat two-dimensional 


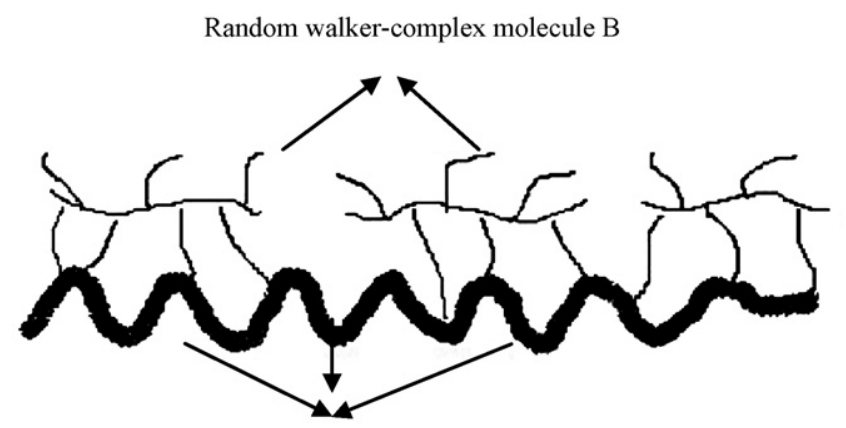

Binding sites $\mathrm{A}$ on particle surface

Fig. 1. Schematic illustration of diffusion-adsorption process on fractal particle surface.

plane, and when the irregularity of the surface is greater, the binding sites A on particle surface and active groups on complex organic molecule B distribute randomly on the lattices of particle surface [13]. The diffusion of organic molecules on the binding sites of particle surface is "a random walking process". When the complex organic molecules randomly walk to the places occupied by the binding sites, the adsorption reaction will happen immediately. At the time of $t$, the density $\rho(t)$ of active groups on complex organic molecule is expressed as $[15,25,26]$ :

$\rho(t)=\rho_{0}[1-p(t)]$

where $\rho_{0}$ is the initial density of active groups in complex organic molecule $(t=0), p(t)$ the reaction probability between active groups of complex organic molecule and the binding sites of particle surface at the time of $t$, and then $[1-p(t)]$ is the nonreaction probability of complex organic molecule at the same time. Eq. (9) can be differentiated to

$$
\frac{\mathrm{d} \ln [\rho(t)]}{\mathrm{d} t}=\frac{1}{1-p(t)} \frac{\mathrm{d} p(t)}{\mathrm{d} t}
$$

For convenience, the complex relation between reaction rate coefficient $k(t)$ and reaction probability $p(t)$ is expressed as

$k(t)=\frac{1}{1-p(t)} \frac{\mathrm{d} p(t)}{\mathrm{d} t}$

Hence

$\frac{\mathrm{d} \rho(t)}{\mathrm{d} t}=-k(t) \rho(t)$

If $S(t)$ is the number of distinct lattice sites visited by complex organic molecules walking randomly at time $t$, the relation between $p(t)$ and $S(t)$ can be expressed as [25,26]:

$$
\begin{aligned}
1-p(t)= & \left(1-\frac{N_{\mathrm{A}}^{\prime}}{N}\right) \times\left(1-\frac{N_{\mathrm{A}}^{\prime}}{N-1}\right) \times \cdots \\
& \times\left\{1-\left[\frac{N_{\mathrm{A}}^{\prime}}{N-S(t)+1}\right]\right\}
\end{aligned}
$$

where $N$ is the total number of distinct lattice sites, $N_{\mathrm{A}}$ the total number of distinct lattice sites that is occupied by surface binding sites and $N_{\mathrm{A}}^{\prime}$ is the number of distinct lattice sites not covered by complex organic molecules at time $t$.
If $S(t) \ll N$, Eq. (13) can be simplified as

$p(t)=\left(1-\frac{N_{\mathrm{A}}^{\prime}}{N}\right)^{S(t)} \quad$ or $\quad p(t)=(1-\alpha \gamma)^{S(t)}$

where $\alpha=N_{\mathrm{A}}^{\prime} / N_{\mathrm{A}}$ and $\gamma=N_{\mathrm{A}} / N$.

If $\alpha \gamma \ll 1$, Eq. (13) can also be simplified as

$1-p(t)=\mathrm{e}^{[-\alpha \gamma S(t)]}$

Hence

$p(t)=1-\mathrm{e}^{[-\alpha \gamma S(t)]}$

Substituting Eq. (15) or Eq. (16) into Eq. (11) gives another equation:

$k(t)=\alpha \gamma\left[\frac{\mathrm{d} S(t)}{\mathrm{d} t}\right]$

Eq. (17) can be written as

$k(t) \propto \frac{\mathrm{d} S(t)}{\mathrm{d} t}$

As suggested by Kopelman [15,17] and Alexander and Orbach [18], for the bimolecular elementary reaction between each active group of complex organic molecule and each surface binding site, the $S(t)$ or $k(t)$ can be expressed as

$S(t) \propto t^{d_{\mathrm{s}} / 2}$

$k(t) \propto t^{\left(d_{\mathrm{s}} / 2\right)-1}$

While based on classical reaction thermodynamics, the adsorption reaction between the complex organic molecules and the binding sites on particle can be written as

$\mathrm{B}+m \mathrm{~A} \rightarrow \mathrm{B}-m \mathrm{~A}$

where $\mathrm{B}-m \mathrm{~A}$ is the product of above reaction. If Eq. (21) obeys the rule of elementary reaction kinetics, the differential reaction rate equation can be expressed as

$-\frac{\mathrm{d}[\mathrm{B}]}{\mathrm{d} t}=k_{2}(t)[\mathrm{A}]^{m} \times[\mathrm{B}]$

where $k_{2}(t)$ is reaction rate constant, which is dependent on time $t$, [B] the concentration of complex organic matter and $[\mathrm{A}]$ is the concentration of binding sites on particle surface.

In general, only [B] is used in adsorption kinetic equations when modeling the adsorption kinetics of Eq. (21) in experiments. Therefore, Eq. (22) can be written as

$-\frac{\mathrm{d}[\mathrm{B}]}{\mathrm{d} t}=k_{1}(t)[\mathrm{B}]$

where $k_{1}(t)$ is the reaction rate constant, dependent on time $t$ :

$k_{2}(t)[\mathrm{A}]^{m}=k_{1}(t)$

The adsorption reaction between the active groups of complex organic molecules and the binding sites on particle surface is expressed as

$\mathrm{B}_{i}+\mathrm{A} \rightarrow \mathrm{B}_{i}-\mathrm{A}$ 
where $\mathrm{B}_{i}$ is the active group of complex organic molecule and $\mathrm{B}_{i}-\mathrm{A}$ is the product of the above reaction. Based on the elementary reaction kinetics, the differential rate equation can be given as

$-\frac{\mathrm{d}\left[\mathrm{B}_{i}\right]}{\mathrm{d} t}=k^{\prime}(t)[\mathrm{A}] \times\left[\mathrm{B}_{i}\right]$

where $k^{\prime}(t)$ is the reaction rate constant, dependent on time $t ;\left[\mathrm{B}_{i}\right]$ is the concentration or density of the active groups of complex organic molecule, equaling to $\rho(t)$.

Eq. (26) can also be written as

$-\frac{\mathrm{d}\left[\mathrm{B}_{i}\right]}{\mathrm{d} t}=k(t)\left[\mathrm{B}_{i}\right]$

Because of both Eqs. (23) and (26) depict the same adsorption reaction process, so the differential rate has the following relationship:

$-\frac{\mathrm{d}[\mathrm{B}]}{\mathrm{d} t} \propto-\frac{\mathrm{d}\left[\mathrm{B}_{i}\right]}{\mathrm{d} t}$

The concentration of complex organic molecule is proportional to the concentration of the active groups of complex organic molecule:

$[\mathrm{B}] \propto\left[\mathrm{B}_{i}\right]$

Based on Eqs. (21)-(29) and power analysis, the following relationship can be obtained:

$k_{1}(t)=\left[k^{\prime}(t)[\mathrm{A}]\right]^{m} \propto k_{2}(t)[\mathrm{A}]^{m}$

Hence

$k_{1}(t)=[k(t)]^{m} \propto k_{2}(t)[\mathrm{A}]^{m}$

In Eq. (23), $k_{1}(t)$ is the instantaneous rate coefficient, and can also be expressed as Eq. (5).

Substituting Eq. (5) into Eq. (31) gives the following relationships:

$[k(t)]^{m} \propto t^{-h}$

$k(t) \propto t^{-h / m}$

Substituting Eq. (20) into Eq. (33) gives the following relationship:

$-\frac{h}{m}=\frac{d_{\mathrm{s}}}{2}-1$

Hence, the spectral dimension $d_{\mathrm{s}}$ for fractal objects is expressed as

$d_{\mathrm{s}}=2\left(1-\frac{h}{m}\right)$

Substituting Eq. (3) into Eq. (35) gives the relationship between the spectral dimension $d_{\mathrm{s}}$ and surface fractal dimension $D_{\mathrm{s}}$ :

$d_{\mathrm{s}}=2\left(1-\frac{h}{K a_{0}^{\left(D_{\mathrm{s}} / 2\right)-1}}\right)$

where $K$ is a proportional coefficient.
Substituting Eq. (36) into Eq. (8) gives the following relationship:

$$
\frac{D}{d_{\mathrm{w}}}=\left(1-\frac{h}{K a_{0}^{\left(D_{\mathrm{s}} / 2\right)-1}}\right)
$$

\section{Materials and methods}

\subsection{Adsorbents}

The GAC of ZJ15 model was selected as adsorbent to adsorb dye compounds. It was purchased from Taiyuan Xinhua Chemicals Plant with 10-20 meshes of particle size. It was purified by distilled water, and then heated for $12 \mathrm{~h}$ in thermostat drying oven at $103 \pm 2{ }^{\circ} \mathrm{C}$.

\subsection{Adsorbates}

Three kinds of dye compounds, direct fast light turquoise blue GL, direct scarlet 4BE, and pink red were selected as adsorbates to investigate the fractal adsorption characteristics of GAC. These dyes were of analytical grade and were used without further purification. These dye compounds are of some typical persistent organic pollutants (POPs) in wastewater treatment. GAC adsorption is an effective way for dye removal from wastewater. The dye index number of direct fast light turquoise blue GL, direct scarlet 4BE, and pink red is C.I. Direct Blue 86 (74180), C.I. Direct Red 2 (23500), and C.I. Acid Red 52 (45100), respectively. The structures of these dyes could be obtained from the handbook of commercial dyes.

Kier and Hall [27] presented the conception of molecular connectivity, and developed the calculation methods for molecular connectivity indices $\left({ }^{n} \chi\right): n$ is the order of $\chi \cdot{ }^{1} \chi$ is the first order term of molecular connectivity index. Sabljic $[28,29]$ found the good linear correlation between ${ }^{1} \chi$ and TSA for 44 organic compounds.

To calculate ${ }^{n} \chi$, the hydrogen-suppressed graph, graph vertex, valence of vertex or degree of vertex and sub-graph from the molecule structure graph should be obtained. Then the ${ }^{n} \chi$ is the sum of sub-graphs with different connection forms [27]. The first order term of sub-graph has one side, and the calculation equation of ${ }^{1} \chi$ is [27,30]:

${ }^{1} \chi=\sum_{i=1, j=1}^{n_{\mathrm{e}}}\left(\delta_{i} \delta_{j}\right)^{-1 / 2}$

where $n_{\mathrm{e}}$ is side number and $\delta_{i}, \delta_{j}$ are the valences of graph vertex of a side in the hydrogen-suppressed graph.

Total surface area, TSA of organic molecule has good relation with the first order term of molecular connectivity index $\left({ }^{1} \chi\right)$, and the equation is [30]:

$\mathrm{TSA}=24.6\left({ }^{1} \chi\right)+57.7 \quad(r=0.956)$

Approximately, the relations of TSA with cross-sectional area $\alpha_{0}$ and the radius of gyration $r_{0}$ of organic molecule are

$\mathrm{TSA}=4 \alpha_{0}, \quad \mathrm{TSA}=4 \pi r_{0}^{2}$ 
Table 1

The molecular characteristics of three dye compounds

\begin{tabular}{lllll}
\hline Dye compound & ${ }^{1} \chi$ & TSA $\left(\mathrm{nm}^{2}\right)$ & $\alpha_{0}\left(\mathrm{~nm}^{2}\right)$ & $r_{0}(\mathrm{~nm})$ \\
\hline $\begin{array}{l}\text { Direct fast light } \\
\text { turquoise blue GL }\end{array}$ & 15.9581 & 1.1257 & 0.2814 & 0.2994 \\
$\quad$ & & & & \\
Direct scarlet 4BE & 16.3989 & 1.1528 & 0.2882 & 0.3030 \\
Pink red & 12.2512 & 0.8977 & 0.2244 & 0.2673 \\
\hline
\end{tabular}

Then the calculated ${ }^{1} \chi$ values of direct fast light turquoise blue GL, direct scarlet 4BE and pink red were 15.9581, 16.3989 and 12.2512 through Eq. (38), respectively. The TSA values were obtained through Eq. (39) and $\alpha_{0}$ or $r_{0}$ values can be determined with the aid of Eq. (40). The results were given in Table 1.

\subsection{Adsorption procedures}

The dye adsorption isotherms in water solutions were obtained by the immersion method. These three kinds of dyes were dried at $105^{\circ} \mathrm{C}$ for $24 \mathrm{~h}$ to remove moisture before use. All of the dye solutions were prepared with distilled water. For adsorption experiments, $1 \mathrm{~g}$ GAC was added into $50 \mathrm{ml}$ dye solutions at the target concentrations. The ionic strength was adjusted with $\mathrm{NaNO}_{3}$ solution to maintain a constant value of $0.01 \mathrm{moll}^{-1}$. $\mathrm{NaN}_{3}$ of $0.02 \%$ (w/w) was added for sterilization. The adsorption was conducted on a reciprocating shaker at $25^{\circ} \mathrm{C}$. The preliminary experiments revealed that the times required reaching equilibrium for direct fast light turquoise blue GL, direct scarlet 4BE and pink red were about 18,5 , and $2 \mathrm{~h}$, respectively. The solution and solid phase were separated by centrifugation at $10,000 \mathrm{rpm}$ for $30 \mathrm{~min}$. A $15-\mathrm{ml}$ aliquot of supernatant was taken and analyzed for dye concentration by a spectrophotometer (Model 7220-S, Shanghai, China) at wavelengths of 622, 535 and $498 \mathrm{~nm}$, respectively. For the adsorption kinetic experiments, the amount of adsorbed dye was determined by analyzing the dye concentration at appropriate time intervals. The adsorption and control experiments were all replicated three times to minimize operational errors.

\section{Results and discussion}

\subsection{Adsorption equilibrium and fractal adsorption isotherm}

Fig. 2 shows the adsorption isotherms of three dyes onto GAC. The adsorption capacity for pink red is the largest, and that for direct fast light turquoise blue GL is larger than that for direct scarlet 4BE. The adsorption capacity difference among the three dyes may be ascribed to their different molecular configuration. As listed in Table 1, molecular diameter of pink red is the smallest, and that of direct fast light turquoise blue GL is smaller than that of direct scarlet 4BE.

Eq. (1) is used to fit the experimental data. The fitting parameters and the average number of binding sites covered by one dye molecule $m$ are presented in Table 2. It can be seen that Eq. (1) fits well with the adsorption isotherm data and one dye molecule can cover more than 3.5 sites on GAC surfaces.

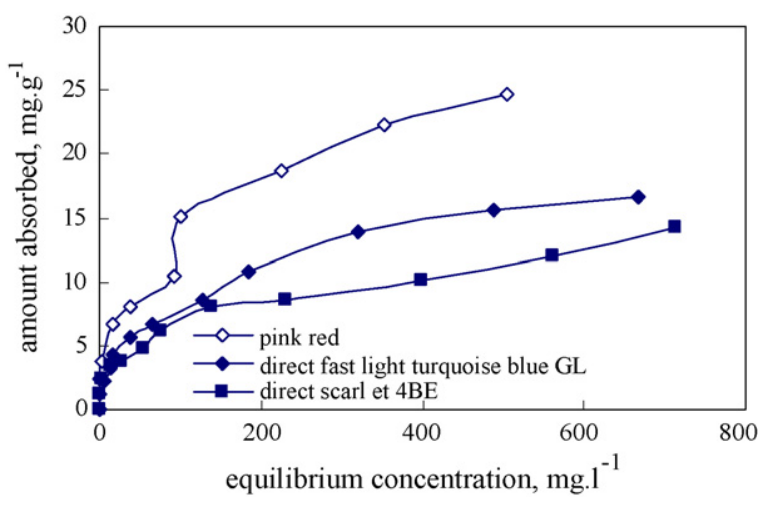

Fig. 2. Adsorption isotherms of dye compounds.

Table 2

Fractal adsorption parameters of three dye compounds

\begin{tabular}{llll}
\hline Dye compounds & \multicolumn{3}{l}{ Fractal Langmuir isotherm } \\
\cline { 2 - 4 } & $1 / m$ & $m$ & $R^{2}$ \\
\hline Direct fast light turquoise blue GL & 0.2734 & 3.6576 & 0.9542 \\
Direct scarlet 4BE & 0.2537 & 3.9417 & 0.9881 \\
Pink red & 0.2834 & 3.5286 & 0.9857 \\
\hline
\end{tabular}

The evaluation of $D_{\mathrm{s}}$ from Eqs. (1), (3) and (4) using the data in Table 2 is illustrated in Fig. 3. The calculated $D_{\mathrm{s}}$ value is 2.59. However, the correlation coefficient of 0.6957 indicates that these data are not in good linear relationship partly due to the lack of data point. If more dyes are utilized, the regression in Fig. 3 could have higher correlation coefficient and more accurate $D_{\mathrm{s}}$ value could be obtained. On the other hand, Avnir et al. [23] and Avnir [24] pointed out that good linear correlation could be achieved if similar absorbate molecules are used.

\subsection{Fractal-like adsorption kinetics}

The adsorption rate coefficient at different time can be obtained by

$\ln \frac{\Gamma_{1}}{\Gamma_{2}}=k_{1}\left(t_{1}-t_{2}\right)$

where $\Gamma_{1}$ and $\Gamma_{2}$ are the amount of solute absorbed $\left(\mathrm{mg} \mathrm{g}^{-1}\right)$ at time $t_{1}$ and $t_{2}$, respectively. It should be pointed out that the rate coefficient discussed here is different from that in classical

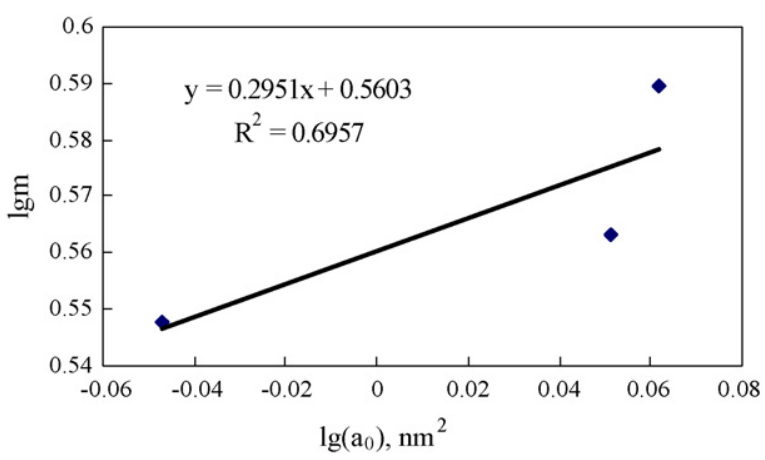

Fig. 3. Linear relation between $m$ and $\alpha_{0}$. 


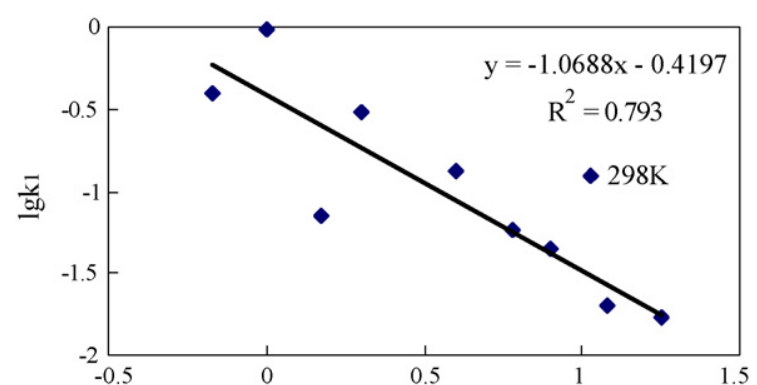

(a)

$\lg (\mathrm{t}), \mathrm{hr}$

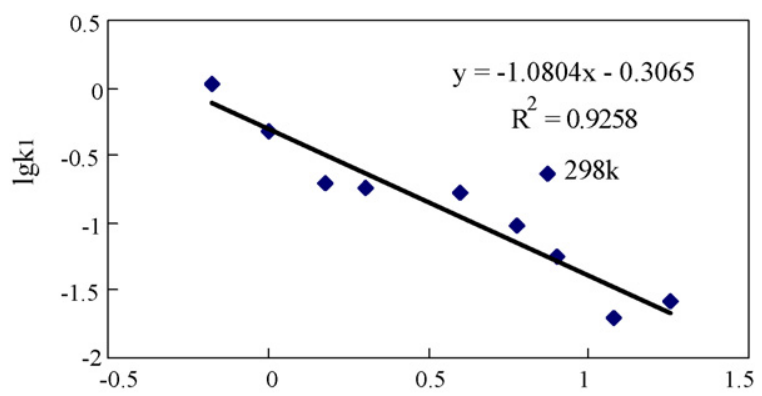

(b)

$\lg (\mathrm{t}), \mathrm{hr}$

Fig. 4. The linear relation between $\lg k_{1}$ and $\lg t$ on absorption of direct fast light turquoise blue GL (a) with initial dye concentration $50 \mathrm{mg}^{-1}$ and (b) with initial dye concentration $100 \mathrm{mg} \mathrm{l}^{-1}$.

Table 3

Kinetic parameters of direct fast light turquoise blue GL adsorbed on GAC $(m=3.6576,298 \mathrm{~K})$

\begin{tabular}{rllll}
\hline Initial dye concentration $\left(\mathrm{mg} \mathrm{l}^{-1}\right)$ & $h$ & $k_{0}$ & $R^{2}$ & $d_{\mathrm{s}}$ \\
\hline 50 & 1.0688 & 0.3805 & 0.793 & 1.42 \\
100 & 0.7723 & 0.1523 & 0.9258 & 1.58 \\
\hline
\end{tabular}

kinetics because the rate coefficient for fractal-like adsorption could be a function of three variables: time, temperature and concentration.

In order to explore the quantitative relationship between rate coefficient and time, the linear regression curves of $\lg k_{1}$ versus $\lg t$ at $298 \mathrm{~K}$ for absorption of direct fast light turquoise blue GL are shown in Fig. 4 (similar results were obtained for the other two dyes, which are not shown). It is evident that the relationship between $k_{1}$ and $t$ can be described by Eq. (41).

Adsorption kinetic data were processed using Eq. (5), and the regression results are listed in Tables $3-5$. It is obvious that most of coefficients $\left(R^{2}\right)$ of linear regression equations is more than 0.75. It indicates that Eq. (5) is appropriate to describe the quantitative relationship between the instantaneous adsorption rate coefficient and time, and the adsorption kinetics of dyes onto GAC is fractal-like.

Table 4

Kinetics parameters of direct scarlet 4BE adsorbed on GAC $(m=3.9417,298 \mathrm{~K})$

\begin{tabular}{lllll}
\hline Initial dye concentration $\left(\mathrm{mg}^{-1}\right)$ & $h$ & $k_{0}$ & $R^{2}$ & $d_{\mathrm{s}}$ \\
\hline 50 & 1.8137 & 0.31 & 0.7936 & 1.08 \\
100 & 2.5145 & 0.6145 & 0.7661 & 0.72 \\
\hline
\end{tabular}

Table 5

Kinetics parameters of pink red adsorbed on GAC $(m=3.5286,298 \mathrm{~K})$

\begin{tabular}{lllll}
\hline Initial dye concentration $\left(\mathrm{mg}^{-1}\right)$ & $h$ & $k_{0}$ & $R^{2}$ & $d_{\mathrm{S}}$ \\
\hline 50 & 1.7742 & 4.2806 & 0.885 & 0.99 \\
100 & 1.0698 & 0.2967 & 0.8694 & 1.39 \\
\hline
\end{tabular}

Since most of $h$ values are larger than one in Ref. [15], the spectral dimension $d_{\mathrm{s}}$ and the order of reaction could not be obtained by using Eqs. (6) and (7). However, using Eq. (35) and $m$ values in Table 1 , the spectral dimension $d_{\mathrm{S}}$ can be calculated at $298 \mathrm{~K}$, and the results are given in Tables 3-5. As shown in Tables 3-5, all the spectral dimensions $\left(d_{\mathrm{s}}\right)$ are less than 2 , which is in agreement with the point of view from Alexander and Orbach [18]. The $h$ or $d_{\mathrm{s}}$ values obtained from above study demonstrate the adsorption reaction kinetics process on GAC surface is fractal-like under dimensional constraints, and show some degree of local heterogeneity in the GAC surface reaction. The convection stirring cannot always be achieved for adsorption reaction in the surface of GAC. In the absence of convective stirring, the diffusive stirring (called self-stirring) is the main form for mass transfer and surface adsorption reaction. The $h$ or $d_{\mathrm{s}}$ values also imply that reciprocating shake is not always effective to eliminate the dimensional constraints. With the reduction of the dimensional constraints, the $d_{\mathrm{s}}$ value should decrease accordingly.

\subsection{Order of fractal-like adsorption kinetics}

Eq. (36) can be written as

$h=K a_{0}^{\left(D_{\mathrm{s}} / 2\right)-1}\left(1-\frac{d_{\mathrm{s}}}{2}\right)$

For the reaction of Eq. (21), substituting Eq. (5) into Eq. (23) gives the following relationship:

$-\frac{\mathrm{d}[\mathrm{B}]}{\mathrm{d} t}=k_{0} t^{-h}[\mathrm{~B}]$

If $t=0,[\mathrm{~B}]=\left[\mathrm{B}_{0}\right]$, Eq. (43) can be expressed as

$\ln [\mathrm{B}]-\ln [\mathrm{B}]_{0}=-\frac{k_{0} t^{1-h}}{1-h}$

or

$t=\left(\frac{h-1}{k_{0}} \ln [\mathrm{B}]\right)^{1 /(1-h)}$

Substituting Eq. (45) into Eq. (43), the following relationship is given

$-\frac{\mathrm{d}[\mathrm{B}]}{\mathrm{d} t}=(h-1)^{-h /(1-h)} k_{0}^{1 /(1-h)}[\mathrm{B}](\ln [\mathrm{B}])^{-h /(1-h)}$

Eq. (46) can be expressed as

$-\frac{\mathrm{d}[\mathrm{B}]}{\mathrm{d} t}=k_{3}(h)[\mathrm{B}]^{x}$

where $k_{3}(h)$ is a function of $h$ :

$k_{3}(h)=(h-1)^{-h /(1-h)} k_{0}^{1 /(1-h)}$ 
$x$ is the order of reaction, and is given as

$x=1+\frac{-K a_{0}^{\left(D_{\mathrm{s}} / 2\right)-1}\left(1-d_{\mathrm{s}} / 2\right)(\ln \ln [\mathrm{B}])}{\left(1-K a_{0}^{\left(D_{\mathrm{s}} / 2\right)-1}\left(1-d_{\mathrm{s}} / 2\right)\right)(\ln [\mathrm{B}])}$

$x$ is the effective order of reaction, which is associated with surface irregularity, the degree of local heterogeneity and absorbate concentration. At most times, $x$ is a fractional number, not an integer, which is one of the characteristics of diffusioncontrolled adsorption reactions. Additionally, $x$ varies with the absorbate concentration, that is, $x$ is not a fixed value during the adsorption process.

\section{Conclusions}

Fractal Langmuir adsorption isotherm was employed to fit the micro-interface adsorption data. The exponent $m$ in the equation is a function of surface fractal dimension $\left(D_{\mathrm{s}}\right)$ and cross-section area $\left(\alpha_{0}\right)$ or radius $\left(r_{0}\right)$ of absorbate molecule: $m \propto \alpha_{0}^{\left(D_{\mathrm{s}} / 2\right)-1} \propto$ $r_{0}^{D_{2}-2}$, while $\alpha_{0}$ and $r_{0}$ can be estimated according to the relation between the first order term of molecular connective index $\left({ }^{1} \chi\right)$ and the total surface molecular area (TSA). Based on the modeling results of dye adsorption onto GAC, $D_{\mathrm{s}}$ of the GAC surface was estimated to be 2.59, indicating the fractal characteristic of GAC surface.

As to the adsorption kinetics of complex molecules onto fractal surfaces, the relations among the parameters of $m, h$ and $d_{\mathrm{s}}$ were developed, and the relation between $d_{\mathrm{s}}$ and $D_{\mathrm{s}}$ was achieved:

$d_{\mathrm{s}}=2\left(1-\frac{h}{m}\right)$

$d_{\mathrm{s}}=2\left(1-\frac{h}{K a_{0}^{\left(D_{\mathrm{s}} / 2\right)-1}}\right)$

The effective reaction order $x$ was related to $d_{\mathrm{s}}, D_{\mathrm{s}}$ and absorbate concentration:

$x=1+\frac{-K a_{0}^{\left(D_{\mathrm{s}} / 2\right)-1}\left(1-d_{\mathrm{s}} / 2\right)(\ln \ln [\mathrm{B}])}{\left(1-K a_{0}^{\left(D_{\mathrm{s}} / 2\right)-1}\left(1-d_{\mathrm{s}} / 2\right)\right)(\ln [\mathrm{B}])}$

Dye adsorption kinetic results showed that the kinetic process had fractal-like characteristic. The instantaneous rate coefficient had a power-law relation with time. Although the calculated $h$ values were often larger than one in this study, both the fractal spectrum dimension $\left(d_{\mathrm{s}}\right)$ and the order of reaction were obtained, and the corresponding $d_{\mathrm{s}}$ values were less than two. The $h$ or $d_{\mathrm{s}}$ values implied that the reciprocating shake was not always effective to eliminate the dimensional constraints.

\section{Acknowledgements}

This research was supported by Grants 20407004, 50178009, 10372112 from National Natural Science Foundation of China, Grant 91078 from Fok-YingTung Education Foundation of Ministry of Education of PR China and Grant from Beijing Municipal Commission of Education.

\section{References}

[1] H.X. Tang, Environmental nano-pollutants and micro-interface water quality process, Acta Scientiae Circumstantiae 23 (2003) 146-155 (in Chinese).

[2] C.P. Chu, D.J. Lee, J.H. Tay, Floc model and intrafloc flow, Chem. Eng. Sci. 60 (2005) 565-575.

[3] B.B. Mandelbrot, The Fractal Geometry of Nature, Freeman, San Francisco, 1982.

[4] J. Feder, Fractals, Plenum Press, New York, 1988.

[5] D. Avnir, D. Farin, P. Pfeifer, Surface geometric irregularity of particulate materials: the fractal approach, J. Colloid Interf. Sci. 103 (1985) 112-123.

[6] P. Pfeifer, M. Obert, Fractals: basic concepts and terminology, in: D. Avnir (Ed.), The Fractal Approach to Heterogeneous Chemistry, Wiley, New York, 1989.

[7] J.C. Russ, Fractal Surfaces, Plenum Press, New York, 1994.

[8] J.F. Douglas, How does surface roughness affect polymer-surface interactions, Macromolecules 22 (1989) 3707-3716.

[9] D. Avnir, D. Farin, P. Pfeifer, Chemistry in noninteger dimensions between two and three. II. Fractal surfaces of adsorbents, J Chem. Phys. 79 (1983) 3566-3571.

[10] P. Pfeifer, M. Obert, M.W. Cole, Fractal BET and FHH theories of adsorption: a comparative study, in: Proceedings of the Royal Society of London. Series A, Mathematical and Physical Sciences, vol. 423, no. 1864, May 8, 1989, pp. 169-188, fractals in the natural sciences.

[11] J.J. Fripiat, L. Gatineau, H. Van Damme, Multilayer physical adsorption on fractal surfaces, Langmuir 2 (1986) 562-567.

[12] Z.F. Ma, Y.L. Jin, Y.M. Liu, H.Q. Yao, The fractal BET model of adsorption, J. Chem. Eng. Chin. Univ. 3 (1994) 288-291.

[13] F. Kanô, I. Abe, H. Kamaya, I. Ueda, Fractal model for adsorption on activated carbon surfaces: Langmuir and Freundlich adsorption, Surf. Sci. 467 (2000) 131-138.

[14] X. Zhao, Y.L. Wang, J.L. Guo, H.R. Han, M.S. Xie, Modification of the micro-interface adsorption model on particles with fractal theoryLangmuir, Freundlich and surface complexation adsorption model, Acta Scientiae Circumstantiae 25 (2005) 52-57 (in Chinese).

[15] R. Kopelman, Fractal reaction kinetics, Science 241 (1988) 1620-1626.

[16] P.W. Klymko, R. Kopelman, Fractal reaction kinetics: exciton fusion on clusters, J. Phys. Chem. 87 (1983) 4565-4567.

[17] R. Kopelman, Rate processes on fractals: theory, simulations, and experiments, J. Stat. Phys. 42 (1986) 185-200.

[18] S. Alexander, R. Orbach, Density of states on fractals: fractons, J. Phys. Lett. 43 (1982) L625-L631.

[19] A. Seri-Levy, D. Avnir, Kinetics of diffusion-limited adsorption on fractal surfaces, J. Phys. Chem. 97 (1993) 10380-10384.

[20] M. Giona, M. Giustiniani, Adsorption kinetics on fractal surfaces, J. Phys. Chem. 100 (1996) 16690-16699.

[21] C.L. Liu, L.J. Xu, X.F. Xian, Fractal-like kinetic characteristics of salt dissolution in water, Colloid Surf. A: Physicochem. Eng. Aspects 201 (2002) 231-235.

[22] M. Kinoshita, M. Harada, Y. Sato, K. Tsubata, Y. Nakamura, Fractal-like behavior of a mass-transport process, AIChE J. 43 (1997) 2187-2193.

[23] D. Avnir, D. Farin, P. Pfeifer, Molecular fractal surfaces, Nature 308 (1984) 261-263.

[24] D. Avnir, The Fractal Approach to Heterogeneous Chemistry: Surfaces, Colloids, Polymers, Wiley, New York, 1989.

[25] R. Kopelman, P. Argyrakis, Diffusive and percolative lattice migration: excitons, J. Chem. Phys. 72 (1980) 3053-3060.

[26] J. Hoshen, R. Kopelman, Exciton percolation. I. Migration dynamics, J. Chem. Phys. 65 (1976) 2817-2823.

[27] L.B. Kier, L.H. Hall, Molecular Connectivity in Structure-Activity Analysis, Academic Press, New York, 1986.

[28] A. Sabljic, On the prediction of soil sorption coefficients of organic pollutants from molecular structure: application of molecular topology model, Environ. Sci. Technol. 21 (1987) 358-366.

[29] A. Sabljic, Predictions of the nature and strength of soil sorption of organic pollutants by molecular topology, J. Agric. Food Chem. 32 (1984) 243-246.

[30] L.S. Wang, Organic Pollution Chemistry (Series B), Science Press, Beijing, 1991 (in Chinese). 